\title{
La maduración del tercer molar y el diagnóstico de la edad. Evolución y estado actual de la cuestión.
}

\author{
Third molar maturation and age assessment. Evolution and \\ state of the art.
}

\section{JL. Prieto 1}

\section{RESUMEN}

La demanda de estimaciones de edad en jóvenes indocumentados está en continuo crecimiento e implica la necesidad de proteger los derechos de los menores reconocidos en los diferentes tratados y acuerdos internacionales cuando un menor es sometido a la acción de la justicia o simplemente solicitan asilo en un país extraño.

En gran parte de los casos el interés se centra en determinar la mayoría o minoría de edad de la persona en cuestión, que está situada en muchos países en los 18 años.

En estas situaciones, la estimación de la edad debe ser lo más fiable y segura posible recomendándose el uso de métodos morfológicos basados en el examen radiológico del desarrollo esquelético o dental.

En los últimos años ha tenido lugar una proliferación de estudios centrados en la maduración del tercer molar como método de estimación de la edad. El objetivo es obtener datos que nos permitan comprender mejor los factores que influyen en el desarrollo madurativo de este diente y establecer valores de referencia más específicos que nos aporten diagnósticos más fiables.

El presente trabajo pretende realizar una revisión de la evolución y estado actual de los conocimientos en este terreno.

Palabras clave: Odontología forense; maduración dental; estimación de la edad; tercer molar; solicitantes de asilo.

Cuad Med Forense 2008; 14(51):11-24

\section{ABSTRACT}

Demand for age assessment of young undocumented people is continually increasing and involves the need to protect the rights of minors recognised in the different international treaties and agreements when those below legal age are subject to legal action or simply seek asylum in a foreign country.

In most of cases, interest focuses on determining the legal age of the person in question, in many countries whether they are 18 years old or younger.

Age assessment must be as reliable and right as possible and the use of morphological methods based on radiological examination of dental and skeletal development is recommended.

In recent years there has been a proliferation of studies focusing on third molar maturation as an age assessment method. The purpose is to obtain data that enable us a better understanding of the factors influencing the maturation of this tooth and establish more specific reference values which provide a more reliable diagnosis.

This paper aims to carry out a brief review about the evolution of knowledge and state of the art in this field.

Key words: Forensic odontology; dental development; age assessment; third molar; asylum seekers.

Correspondencia: Dr. José Luis Prieto. Juzgados de 1ํㅡㄴ Instancia e Instrucción no 2 y nํ 4. 28100 Alcobendas (Madrid). email: prietoaf@med.ucm.es

1 Médico Forense. Odontólogo. 


\section{INTRODUCCIÓN.-}

La identificación de cadáveres o personas vivas es, dada su trascendencia en el ámbito ético y legal (civil o penal) uno de los principales retos a los que los profesionales de la Medicina forense nos enfrentamos cotidianamente.

En particular, cada vez es más habitual la demanda de exámenes médicos de estimación de la edad en jóvenes indocumentados, generalmente inmigrantes, que en unos casos carecen de documentación fiable en la que conste la fecha de nacimiento y en otros la ocultan con el fin de obtener los beneficios que habitualmente se contemplan, en las legislaciones de los países desarrollados, para el menor de edad.

La estimación de la edad en estos casos se plantea ante la necesidad de proteger los derechos de los niños o niñas menores de edad, reconocidos por los distintos convenios y tratados internacionales [18, 20,33] cuando éstos se ven sometidos a la acción de la justicia o simplemente solicitan asilo en un país extraño.

En muchos países, entre ellos España, el interés diagnóstico de la edad en el ámbito penal se centra en los 14, 18 y 21 años. Los menores de 14 años se consideran inimputables (no responsables de sus actos); entre los 14 y los 18 años se aplica de manera específica la Ley del Menor, que prevé un trato especial para quienes cometen algún delito en este rango de edad; y hasta los 2 I años, aun tratándose de mayores de edad, la ley contempla una reducción de la imputabilidad [50].

En los casos de petición de asilo o menores no acompañados, el interés se centra en determinar la mayoría o minoría de edad de la persona en cuestión, situada ésta en muchos países en los 18 años.

En todos estos casos, la estimación de la edad debe ser lo más fiable y segura posible.

En la etapa infantil y juvenil, algunos autores recomiendan el uso de métodos morfológicos basados en el examen radiológico del desarrollo dentario y esquelético [78].

Sin embargo, la metodología empleada es variable y los criterios seguidos internacionalmente sobre la aplicación con carácter oficial de estas técnicas son dispares, argumentando ciertos países la escasa validez que muchos trabajos científicos otorgan actualmente a este tipo de pruebas, cuyos márgenes de error no permiten realizar diagnósticos con la fiabilidad que en estos casos se requiere $[2,3,4,5]$. Es por ello que en algunos países las pruebas radiográficas sólo se utilizan en casos criminales [83]. A pesar de ello, los programas desarrollados por instituciones oficiales y organizaciones no gubernamentales, han establecido protocolos de buenas prácticas en los que se incluyen los elementos correspondientes a la estimación de la edad [I].

El Grupo alemán para el estudio de la edad, considera que el examen dirigido al diagnóstico de edad debería incluir [34]:

- Un examen físico con inclusión de datos antropométricos, signos de maduración sexual y cualquier anomalía relevante del desarrollo en relación con la edad.

- Un examen radiográfico de la mano izquierda.

- Un examen dental con registro del estado de la dentición y evaluación de una ortopantomografía.

Se recomienda que los métodos se utilicen en combinación a fin de incrementar la exactitud en la estimación de la edad [84].

Aunque el tercer molar es el diente más variable, dada la escasez de indicadores alternativos del grado de maduración, la evaluación del grado de maduración de este diente constituye una de las principales herramientas para la estimación de la edad en estos supuestos. 
Debido a ello, en los últimos años han proliferado los estudios centrados en la maduración del tercer molar como método de estimación de la edad, con la finalidad de aportar datos que nos permitan comprender mejor los factores que influyen en el desarrollo madurativo de este diente y establecer valores de referencia más específicos que nos proporcionen diagnósticos más fiables.

El presente trabajo pretende realizar una revisión de la evolución de los conocimientos en este terreno.

\section{DEFINICIÓN DE EDAD.-}

El diccionario de la Real Academia de la Lengua define la edad como el "tiempo que una persona ha vivido, a contar desde que nació". La fecha de nacimiento es, por tanto, el dato a partir del cual se calcula la edad del individuo cuando nos referimos a ella utilizando un criterio cronológico o vital. Pero no es éste el único criterio posible para referirnos a la edad de una persona. Podemos hablar de edad anatómica (grado de desarrollo físico), de edad mental (nivel de desarrollo de la inteligencia que expresa la edad cronológica que corresponde del modo más típico a determinado nivel de rendimiento) e incluso de edad social (grado de madurez alcanzado que permite hacer frente a las exigencias de nuestro entorno social, familiar,...)

La edad tiene una gran importancia en la vida administrativa, civil y penal del individuo. La mayoría de edad significa automáticamente (en ausencia de limitaciones intelectuales) adquirir la plena capacidad de obrar.

Según la legislación española, (artículo 12 de la Constitución y 315 del Código Civil) la mayoría de edad empieza a los dieciocho años cumplidos. La edad legal se establece, por tanto, en base a un criterio estrictamente cronológico. Sitúa al individuo en un contexto temporal variando de forma paralela al transcurso de éste, de forma que la edad es un elemento en continua mutación durante la vida del individuo. Cualquier indicación de la edad de un sujeto, se habrá de realizar con referencia a dos momentos concretos: el del examen (o el de los hechos) y la fecha de nacimiento, establecida habitualmente a partir de los elementos de carácter testimonial y/o documental (certificado o historial médico) que generan la inscripción en el Registro Civil.

Llegados a este punto se hace preciso subrayar la discrepancia de criterio entre el concepto de edad en términos legales y biológicos. Los conceptos de edad cronológica y anatómica no son directamente equiparables pues, mientras la edad cronológica sigue un curso continuo e inexorable (mañana o dentro de un año seremos exactamente un día o un año más viejos que hoy) la edad biológica, determinada a través del grado de maduración de ciertas estructuras anatómicas (esqueleto, dentición,...) presenta una evolución más irregular e inconstante, con aceleraciones, deceleraciones y detenciones, bajo la influencia de diversos factores tanto genéticos como ambientales muchos de ellos imposibles de determinar [ 10,54$]$. Cada niño tiene su propio ritmo de crecimiento y maduración, que no es un simple reflejo de su edad cronológica. Afortunadamente, los estudios científicos pretenden acotar ese rango de variabilidad, dentro de unos márgenes predecibles.

\section{DESARROLLO DENTAL Y ESTIMACIÓN DE LA EDAD.- Erupción dentaria.-}

La edad dental puede ser estimada con precisión en la niñez, dado que en ese periodo muchos dientes se están desarrollando de manera simultánea.

La inspección visual de la erupción dental fue el primer método de estimación de la edad y el más utilizado. En un trabajo titulado "The Teeth a Test of Age", Edwing Saunders propuso en I 837 al Parlamento Inglés utilizar la erupción dental como método para determinar la edad de los 
niños que eran empleados en las fábricas, cuyo límite legal se situaba en los nueve años [9]. Durante mucho tiempo, y aún hoy en día en muchas partes del mundo en que no existen registros de nacimiento, el desarrollo dental se utiliza como un indicador de la edad en niños, adoptándose como criterio biológico-legal.

No obstante, aunque es un método rápido, barato y no muy influenciado por el error intra o interobservador, la erupción no es un buen indicador de la edad cuando se utiliza de forma aislada, debido a ciertos factores como la variabilidad interindividual o poblacional [32, 63], anomalías de origen sistémico o local [93] o el periodo de tiempo en el que no se producen cambios (por ejemplo desde los dos años y medio o tres en que finaliza por término medio la erupción de la dentición decidua hasta los cinco o seis en que comienza la erupción de la permanente) [92].

Numerosos autores han investigado la cronología y secuencia de erupción en diferentes poblaciones [26, 29, 35, 72, 74, 77, 82, 88, 94]. Algunas de estas investigaciones examinan la correlación entre la erupción dentaria y otros parámetros de desarrollo como el estudio de Lewis y Garn [49] que valora, entre otros, parámetros tales como el crecimiento somático y sexual, personalidad y estado de salud, o el de Green [38] que pretende establecer la correlación entre edad dental, esquelética, cronológica, peso y altura, encontrando una mayor correlación entre la edad dental y cronológica, superior a la existente entre la edad dental y la ósea. Hagg y Taranger [4I] estudian la correlación entre la emergencia dentaria y el crecimiento máximo puberal, observando una baja correlación entre los indicadores de desarrollo somático y dental. Baume y cols [ [ I] han demostrado cambios en la erupción dentaria relacionados con los niveles de hormonas hipofisarias.

\section{Maduración dental.-}

La mineralización de las coronas de los dientes deciduos comienza sobre los 3 ó 4 meses de vida intrauterina continuando su calcificación tras el nacimiento durante el periodo neonatal [17]. La formación de las raíces generalmente se completa entre el año y medio y los tres años de edad.

Por su parte, la mineralización de la dentición permanente dura aproximadamente unos nueve años, comenzando con el primer molar permanente en torno al momento del nacimiento [25].

El proceso de maduración dental se correlaciona con diferentes estadios de mineralización que pueden ser observados a través de registros radiográficos y experimenta cambios mucho más uniformes y graduales que la erupción. Está más controlado por factores genéticos y menos influenciado por factores externos que todos los demás criterios mensurables de maduración [49, 94]. Esta es la razón por la que se han desarrollado diversos métodos de estimación dental de la edad.

Todos los métodos de evaluación de la edad basados en la maduración dentaria siguen una misma sistemática. Primero se evalúa el estadio de desarrollo de cada uno de los dientes a partir de registros radiográficos, siendo el método de elección la radiografía panorámica u ortopantomografía (OPT). A continuación, el estadio de desarrollo se relaciona con la edad correspondiente al mismo, derivada del estudio de una muestra de edad conocida. Este método de estimación se basa en evaluaciones subjetivas de los estadios y posee ciertos defectos. Por otra parte ha de tenerse en cuenta la variabilidad biológica en el desarrollo de cada uno de los dientes.

Debido a las diferencias existentes entre métodos y poblaciones de distinto origen, estos elementos han de ser expresados, así como el intervalo de confianza. Numerosos estudios han proporcionado escalas de maduración, tanto en dentición decidua como permanente, en diferentes poblaciones, identificando sucesivos estadios de desarrollo, aunque presentan diferencias en la metodología empleada (métodos longitudinales frente a transversales, definición de los estadios evolutivos,...) $[22,61,62,63,65]$. La mayoría de estos métodos de estimación de la edad presen- 
tan durante el periodo infantil, en que puede observarse el desarrollo simultáneo de varios dientes, variaciones de unos 2 años respecto a la media para intervalos de confianza del 90-95\%, lo que indica una exactitud bastante baja.

Estudios realizados sobre muestras de edades conocidas [5I, 8I], presentan diferencias de unos 6 meses respecto a las edades reales.

Nolla [65] clasificó el desarrollo dentario en 10 estadios de calcificación, desde el estadio de cripta hasta el cierre apical de la raíz. El estudio de Nolla advirtió que el desarrollo de la mineralización comenzaba y finalizaba antes en el sexo femenino, aunque no parecían existir diferencias en la secuencia de finalización del desarrollo. El método de Nolla es uno de los más utilizados en la clínica como procedimiento para la estimación del desarrollo de la dentición permanente. Diversos estudios [ [3, 40, 87], aplicando el método de Nolla encuentran un error medio de estimación, para el $95 \%$ de intervalo de confianza, de unos dos años.

Uno de los sistemas más universalmente utilizados para valorar el grado de desarrollo de la dentición permanente es el propuesto por Demirjian Goldstein y Tanner [22] a partir del análisis de una muestra de niños de origen franco-canadiense. El método original valora el grado de calcificación de los siete dientes de la hemiarcada mandibular izquierda, excluyendo el tercer molar, a partir de registros radiográficos. Se establecen 8 estadios de maduración en cada diente $(\mathrm{A}$ a H), desde el inicio de la calcificación de la corona hasta el cierre apical de la raíz, de manera similar al método de Nolla. Se atribuye a cada diente un estadio de formación, que se convierte en una puntuación, en función del sexo, siguiendo la misma técnica matemática utilizada para la valoración de la maduración esquelética por el método de Tanner-Whitehouse [89]. Se suman las puntuaciones de los siete dientes, obteniendo la denominada puntuación de madurez dentaria en una escala de 0 a I 00. Esta puntuación se transforma, a partir de las tablas correspondientes, en edad dentaria. El método tiene el inconveniente de que no incluye la valoración de los terceros molares, por lo que sólo puede ser utilizado para edades preadolescentes.

Con posterioridad, este mismo autor ha desarrollado actualizaciones del método original, proponiendo un sistema de valoración de cuatro dientes (ambos premolares y molares) con estándares distintos [2I].

En todos los casos, dado que el desarrollo madurativo es diferente en ambos sexos, deberá determinarse el sexo previamente [48].

La gran difusión de este método como procedimiento para estimar la edad en el periodo infantil, ha hecho que los resultados del estudio de Demirjian hayan sido comprobados en otras poblaciones. Numerosos trabajos desarrollados en los últimas décadas demuestran un ligero retraso de la maduración de la población franco-canadiense original, lo que provoca una sobreestimación cuando se aplican los resultados originales de este método a otras poblaciones [14, 19, 24, 28, $46,52,53,56,66,67,75,95]$. Dicha sobreestimación oscila entre unos meses y varios años de edad, recomendándose el uso de estándares basados en estudios realizados sobre la misma población sobre la que se van a aplicar.

Buscando un modelo estadístico más ajustado, que explique lo mejor posible la correlación entre el grado de maduración dental y la edad cronológica, Teivens y Mönstard [68] han desarrollado recientemente diversas funciones matemáticas como modificación al método original de Demirjian, obteniendo los mejores resultados cuando se aplica un modelo de regresión cúbica $(R 2=0,95)$. Este modelo ha sido ensayado en un estudio comparativo entre sujetos de población sueca y coreana, demostrando diferencias estadísticamente significativas, con un desarrollo más temprano en la población sueca, estimado en 2 meses para varones y 6 meses para mujeres [90]. 


\section{EL TERCER MOLAR EN LA ESTIMACION DE LA EDAD.-}

La estimación de la edad se complica una vez se ha producido el cierre apical de las raíces del segundo molar permanente (aproximadamente a los 14 años) debido a la variabilidad que presenta el desarrollo del tercer molar. El tercer molar es el diente con mayor frecuencia de agenesias [3 I] y el más irregular en su secuencia de maduración [44] y, al contrario que en el resto de la dentición, ésta suele ser más precoz en varones que en mujeres [48].

A pesar de ello, el continuo aumento de la inmigración de jóvenes de países del tercer mundo a los países industrializados, y la necesidad de contar con un procedimiento fiable y seguro de estimación de la edad cuando se carece de documentación que acredite la fecha de nacimiento, está impulsando el desarrollo de estudios basados en la maduración del tercer molar desde hace años $[43,59,79,80]$ único diente en proceso de maduración en edades juveniles, especialmente debido a que su grado de mineralización puede ser fácilmente determinado a partir de un método no invasivo como es la radiología.

Los argumentos principales contendidos en los distintos trabajos publicados son los siguientes.

\section{Elección del método adecuado.-}

Para que un método de estimación de la edad se considere adecuado, debería cumplir los siguientes requisitos [78]:

- Debe ser claro y verificable, presentado a la comunidad científica, como regla general, a través de su publicación en revistas arbitradas (peer-reviewed).

- Información clara sobre el grado de exactitud de estimación de la edad.

- El método necesita ser suficientemente preciso para satisfacer las demandas específicas del caso concreto.

- En los casos de estimación de la edad de individuos vivos han de tenerse en consideración los principios de la ética médica y las normas legales, especialmente si se realiza alguna actuación de carácter médico.

La elección de un método concreto dependerá de las condiciones específicas de cada caso y principalmente de la exactitud necesaria.

De los numerosos métodos de valoración del proceso de maduración del tercer molar, parece haber actualmente un amplio consenso en considerar el método de Demirjian [22] el más adecuado por diversas razones:

Los estadios de Demirjian se definen por cambios morfológicos, más objetivos en su valoración que las estimaciones más especulativas de la longitud [69, 70]. Los estadios de formación de la raíz son más fáciles de definir y muestran los valores más altos de concordancia intra e inter-observador y de correlación entre los estadios definidos y la edad real [23].

\section{Material de Referencia.-}

Para ser utilizado como estudio de referencia, éste debería reunir ciertos requisitos [85]:

- Tamaño adecuado de la muestra. El número de sujetos de cada grupo de sexo y edad debería ser diez veces el número de rasgos examinados.

- La edad señalada por los sujetos debería ser verificada.

- Distribución equilibrada de los sujetos en todos los grupos.

- Los datos han de ser recogidos separadamente para ambos sexos.

- Debería registrarse la fecha del examen.

- Los rasgos examinados deberían definirse sin ambigüedades. 
- La técnica utilizada en el examen debería describirse de forma precisa.

- Es indispensable incluir la información sobre el origen genético-geográfico, estatus socioeconómico y estado de salud de la población de referencia.

- Para cada rasgo examinado debería proporcionarse el tamaño muestral, la media y los parámetros estadísticos de desviación.

- Sería deseable incluir la información del error inter e intra-observador.

Meinl et al [57] llaman la atención sobre el cuestionamiento de los términos "origen genético-geográfico", "estatus socio-económico" y "estado de salud", dada la dificultad de establecer la definición de estos parámetros y los aspectos éticos de estos términos, lo que condicionaría su uso de forma confiable en un estudio.

\section{Exactitud y confiablidad. El error estándar.-}

En comparación con otros métodos diagnósticos de la edad, los empleados en la estimación de la mayoría o minoría de edad deberían minimizar los falsos positivos a fin de evitar clasificar erróneamente como mayor de edad a un menor.

La confiabilidad de un método es la condición por la cual una medida y su técnica acompañante son coherentes.

La confiabilidad presenta dos propiedades:

- Precisión (repetición de un resultado). La precisión de un método de determinación de la edad depende de, al menos, tres factores independientes [88]:

o Posibilidad de interpretar y clasificar correctamente los estadios de desarrollo del diente.

- Calidad y aplicabilidad del material de referencia.

o Variabilidad biológica individual de desarrollo.

- Exactitud (cercanía de la estimación a su valor real).

Los trabajos desarrollados revelan una buena precisión, pero una pobre confiabilidad, demostrada por unos coeficientes de correlación bajos y diferencias elevadas entre la edad estimada y la real. Estos rangos de variación son lo suficientemente amplios como para no permitirnos dar una respuesta satisfactoria a la pregunta sobre la mayoría o minoría de edad de un individuo, sobre todo en aquéllos que se encuentran en torno a la edad límite de los 18 años. Los errores intra e inter-observador encontrados son elevados $[23,73,76]$ con una mayor correlación cuando se emplea el método de Demirjian en el tercer molar mandibular [7, 23].

En un estudio realizado por el Research Committee of the American Board of Forensic Odontology, Mincer y cols [60] evalúan la precisión en la estimación de la edad a partir del estado de desarrollo del tercer molar inferior, valorado según el método de Demirjian. Según este estudio, los estadios A a D (hasta la formación completa de la corona) y el estadio $\mathrm{H}$ (cierre completo apical) indicarían con una alta probabilidad que el individuo es menor o mayor de 18 años, respectivamente. El grado de exactitud, establecido en base a la diferencia entre la edad real y la calculada por el grado de desarrollo dentario, se sitúa en 4,8 años para el rango del 95\% (dos desviaciones estándar). Thorson y Hägg [9I] utilizando el mismo sistema de valoración en una muestra de población sueca han observado una pobre relación entre la edad cronológica y la edad dental (infraestimación de la edad cronológica), con una diferencia media entre la edad estimada y cronológica de unos \pm 4.5 años en niñas ( $95 \%$ intervalo de confianza) y \pm 2.8 años en niños, y un error intra-observador de \pm 0.8 años ( $95 \%$ intervalo de confianza) lo que inhabilita para los autores este método para la estimación de la edad. 
Similares resultados obtienen Kullman y cols [47] aplicando un sistema basado en 7 estadios de desarrollo de la raíz, con desviaciones estándar de I a 2 años sobre la edad media.

En la revisión realizada por Rirz-Timme et al [78] el tercer molar arroja unos valores de error estándar (SEE) que varían en un rango de I-2,5 años, con coeficientes de correlación ( $r$ ) entre 0.32 y 0.85 .

El uso del error estándar como medida de la exactitud, ha sido criticado por diversos autores $[8,36]$ y recientes trabajos proponen el uso de la probabilidad Bayesiana como alternativa al análisis de regresión [16] aunque se consideran necesarios más estudios al respecto.

\section{Dimorfismo sexual.-}

Quizás debido a que buena parte del proceso de formación del tercer molar se produce después de alcanzada la pubertad, se observa un dimorfismo sexual contrario al resto de los procesos madurativos, y los varones alcanzan los distintos estadios de desarrollo antes que las mujeres, independientemente de su origen étnico, conclusión en la que coinciden los estudios publicados hasta la fecha [7, 32, 39, 40, 42, 43, 45, 48, 57, 73, 76, 85, 91, 96].

\section{Diferencias topográficas.-}

Los estudios que han evaluado el desarrollo de los terceros molares en la arcada maxilar y mandibular, parecen coincidir en el mayor avance en la maduración de los molares maxilares sobre los mandibulares $[6,14,37,57,58,73,85,96]$. Para Mincer [60] este hecho podría reflejar diferentes mecanismos de control en el proceso de desarrollo de cada arcada. Así mismo observa que la combinación de resultados de los dientes de ambas arcadas parece mejorar ligeramente la exactitud.

Aunque la impactación de los terceros molares se ha esgrimido como causa de retraso en la formación radicular [45], un reciente trabajo de Friedrich [27] concluye que la topografía del tercer molar no influye en el tiempo de desarrollo radicular.

La totalidad de los trabajos coinciden en la ausencia de diferencias significativas en función del lado (izquierdo o derecho) $[57,73,76,96]$.

\section{Influencia de patologías.-}

El desarrollo dental puede alterarse por enfermedades de larga evolución, síndromes congénitos, deficiencias nutricionales o trastornos hormonales, entre otros. Por otra parte, los factores que influyen en la formación dentaria son difíciles de identificar.

Meinl et al [57] llaman la atención sobre las consecuencias de la exclusión de individuos que presentan ciertas patologías o un desarrollo irregular, de forma que los métodos de estimación de la edad sólo sean aplicables a individuos con un estado dental supuestamente normal. En su estudio deciden no realizar exclusiones por estos motivos, incluyendo a todos los individuos de la muestra original, observando que ninguno de los individuos "extremos" poseía características asociadas con un crecimiento anormal y, por otra parte, individuos con severas alteraciones del desarrollo, no mostraban hallazgos "alarmantes". De acuerdo a estos resultados asumen la hipótesis de que el desarrollo dentario se encuentra bajo potentes mecanismos de regulación que parecen difíciles de alterar, incluso bajo condiciones patológicas.

\section{Influencia de los factores étnicos, geográficos y socioeconómicos.-}

Al igual que cualquier otro análisis antropológico, las características de la población de referencia es un elemento significativo. La influencia de factores genéticos, nutricionales y geográficos 
deben ser tenidos en cuenta en el desarrollo de estándares. Es por ello que la aplicación de los estándares generados en grupos étnicos diferentes a la población de referencia ha sido sujeto de controversia.

Hasta ahora se han acometido diversos estudios en diferentes poblaciones, con el ánimo de observar la utilidad del tercer molar como un indicador confiable de la edad [7, 12, 15, 30, 39 , $42,43,47,55,57,59,60,64,68,71,72,73,76,79,85,96,97]$. Estos estudios han demostrado que el desarrollo dental muestra ligeras variaciones entre poblaciones diferentes, haciendo necesario el desarrollo de estudios específicos.

Aunque se observa una cierta heterogeneidad al comparar los resultados de los estudios realizados en distintas poblaciones, los resultados de un trabajo [76] realizado sobre una muestra de 1050 ortopantomografías de jóvenes españoles de 14 a 2 I años, evidencian que la población española muestra un desarrollo madurativo de los terceros molares inferiores más acelerado que la población Norteamericana, Franco-Canadiense, Germana, Austriaca y Escandinava, y más similar a la población Hispana de los Estados Unidos. Otros hallazgos son análogos a los observados en dichas poblaciones, como la maduración más acelerada en los varones respecto a las mujeres, la concordancia cuando se comparan ambas hemiarcadas o el grado de precisión y fiabilidad.

Garamendi y cols [30] han estudiado una muestra de I I 4 varones de origen marroquí, inmigrantes ilegales, cuya edad real fue obtenida con posterioridad. El examen incluyó el estudio radiográfico dental para la estimación de la maduración del tercer molar según el método propuesto por Demirjian y Goldstein ya comentado. Los resultados demuestran que éste constituye un buen método diagnóstico de la edad -aunque se aplicaron los estándares obtenidos del análisis de la población franco canadiense original- aumentando su eficacia al combinarse con los métodos de valoración de la maduración ósea.

La carencia de datos sobre la influencia del factor étnico en la mineralización constituye una restricción en la fiabilidad de la estimación de la edad y por lo tanto del valor de la información forense, esencial para la seguridad jurídica. Ésta ha venido determinada por diversas causas, tales como el uso de diferentes métodos de estimación, pequeños tamaños muestrales, o datos de nacimiento no comprobados para la población africana, lo que impide que los datos sean directamente comparables.

En varios trabajos conjuntos con colegas sudafricanos y japoneses, Olze et al [68, 7I, 72] evalúan la posible influencia del factor étnico en la mineralización y erupción del tercer molar. Observan un proceso de maduración más lento en población mongoloide, que alcanzaría los distintos estadios de mineralización I a 2 años más tarde que los caucasoides, mientras que los africanos lo harían de I a 2 años más pronto. El comportamiento es similar respecto a la erupción. Estos autores consideran que estas discrepancias podrían deberse a la diferencia en las dimensiones del paladar, más pequeño en mongoloides, lo que causaría un retraso en la erupción y, derivado de ello, en la mineralización.

Blankenship y cols [1 2] y Harris [42] evalúan las diferencias entre negros y blancos norteamericanos. Los resultados son coincidentes con los de Olze y cols, al señalar una mayor velocidad de maduración en población negra.

Ahora bien, los estudios científicos desarrollados, aunque suponen una aportación significativa al conocimiento de la maduración del tercer molar, no resuelven el problema de enfrentarnos ante la determinación de la edad de un joven inmigrante indocumentado. En el caso de España y de la mayoría de países europeos, estos inmigrantes son, en proporciones diversas, de origen marroquí, subsahariano, latinoamericano y de la Europa del Este. 
Martín de las Heras y cols [55] comparan el desarrollo del tercer molar en población española procedente del norte de España con población de origen español y marroquí residente en una de las ciudades españolas del norte de África (Ceuta). El interés de este trabajo se centra en el hecho, apuntado anteriormente, de que la población magrebí es uno de los grupos de inmigración ilegal más frecuente en España y otros países de la Europa Mediterránea.

La carencia de estándares de maduración para dichas poblaciones y la dificultad de que se realicen en los países de origen, hace necesario el desarrollo de estudios que nos permitan valorar la forma en que la maduración dentaria tiene lugar en dichas poblaciones y contar con datos más fiables.

Las variaciones inter-poblacionales demostradas por estos estudios ponen de relieve la necesidad de desarrollar estudios específicos.

Se necesitan más investigaciones en esta línea.

\section{La cuestión de la mayoría de edad.-}

Los resultados de los trabajos publicados coinciden en afirmar la idea de que una vez que el tercer molar alcanza el estadio $\mathrm{H}$ de Demirjian, la probabilidad de que el individuo sea mayor de edad supera el 90\%, independientemente del origen étnico, sexo o diente evaluado $[7,57,60,85]$. Por tal motivo se puede considerar este elemento como un marcador útil para resolver la cuestión de si un individuo de edad desconocida puede ser considerado un adulto a efectos legales.

\section{Aspectos éticos.-}

No es habitual en la práctica que se solicite el consentimiento expreso del menor para su exploración y la realización de los exámenes complementarios, incluidas las pruebas radiológicas cuyo uso, salvo en casos penales, no parece justificado.

Dado que el examen va a afectar a su intimidad, y las pruebas radiológicas suponen una exposición radioactiva con un potencial efecto lesivo, entendemos que debe ser obligatorio obtener el consentimiento del explorado, y suspender el examen en caso de faltar éste. Este aspecto viene recogido en la guía de buena práctica del programa de niños no acompañados en Europa [I].

Ha de apreciarse además, la perversión que supone el uso de técnicas que se han desarrollado con objetivos clínicos - evaluar el grado de desarrollo madurativo para una edad determinada -en la búsqueda de posibles patologías, al invertir, con fines periciales, su aplicación- deducir la edad de un individuo a partir de su maduración - en la que no existe una indicación clínica que justifique su uso.

\section{Control de calidad.-}

Son escasos los intentos de poner en común la estandarización, calibración y los procedimientos de evaluación, lo que aboga por la necesidad de unas directrices a seguir sobre estos aspectos.

La dificultad que entraña el diagnóstico y las potenciales fuentes de evaluación hacen, por tanto, necesaria la elaboración de unas guías comunes de actuación, basadas en las evidencias científicas, que unifiquen los criterios a seguir, tales como las del Study Group on Forensic Age Diagnosis (AGFAD), fundado en Berlin en el año 2000. El grupo ha publicado directrices sobre el diagnóstico de edad en vivos para casos criminales, civiles y procedimientos de asilo, entre otros [84]. 
La Organización Internacional de Odonto-Estomatología Forense (IOFOS) ha publicado procedimientos recomendados para asegurar la calidad en la estimación forense de la edad http://www.odont.uio.no/foreninger/iofos/quality/Age-IOFOS.htm.

En relación a estas recomendaciones, Solheim and Vonen [86] resaltan las discrepancias de criterio entre expertos sobre los pasos a seguir, divididos entre aquéllos que sólo quieren aplicar un método estadístico e informar de los resultados y los que prefieren expresar la opinión del experto tomando en consideración las condiciones de vida de las personas, tales como estado de salud y nutrición, hallazgos clínicos y, por supuesto los resultados de los métodos estadísticos científicos. Estos autores coinciden con el German Interdisciplinary Working Group for Age Diagnostic [34] en que las conclusiones deberían finalizar con una evaluación de la edad cronológica más probable.

Tal como recomiendan Ritz-Timme y cols [78] deben realizarse esfuerzos para el desarrollo de controles externos de calidad, algo perfectamente posible en el terreno de la estimación de la edad, a fin de garantizar estándares de calidad que permitan responder adecuadamente al importante papel que en el terreno legal y social juega la medicina forense en relación al diagnóstico de la edad.

\section{CONSIDERACIONES FINALES.-}

La cuestión de los informes periciales sobre la edad de jóvenes indocumentados es un reto de primer orden que exige tomar conciencia de su complejidad.

Es ampliamente conocido por los expertos médicos que la estimación de la edad es un procedimiento inexacto y aproximado. Es imposible tener la certeza de la edad de un individuo y se constata un considerable margen de error de un mínimo de 20 a 24 meses por exceso o defecto.

Las recomendaciones de UNHCR constatan que [I]:

Cuando se utilizan procedimientos científicos en la determinación de la edad debe proporcionarse el margen de error.

Tales métodos deben ser seguros y respetuosos con la dignidad humana.

El valor de una estimación depende de la pericia y experiencia del profesional médico que realiza el examen. Tales estimaciones se basan en una variedad de factores entre los que se incluyen: examen físico y constatación de estatura, peso y presencia de caracteres sexuales secundarios; maduración psicológica; examen dental y análisis de la maduración ósea. Ninguno de estos factores en solitario ha comprobado tener una validez concluyente para el establecimiento exacto de la edad. Además las diferencias culturales, raciales y étnicas pueden variar considerablemente los indicadores de edad.

Se debe insistir en respetar los márgenes de error de los métodos estadísticos, sin forzar las interpretaciones para conseguir una respuesta categórica a la cuestión planteada. Debe hacerse hincapié en el carácter parcial y complementario de las pruebas biológicas, con el fin de que no se desestime por principio o por ahorro de energía procesal la búsqueda de datos testimoniales o documentales que ayuden a datar adecuadamente al sujeto.

Sólo con todos los datos a la vista podrá el Juzgador tomar una decisión sobre la mayoría de edad del presunto menor, teniendo en cuenta los aspectos médicos, que son una parte del problema, y los no médicos, incluyendo la valoración que en cada caso merezcan las situaciones de duda, teniendo en cuenta el contexto procesal en que se encuentren. 


\section{BIBLIOGRAFÍA.-}

I. UNHCR, Guidelines on Policies and Procedures in dealing with Unaccompanied Children Seeking Asylum, Geneva 1997

2. UNHCR NEWSLETTER. Separated Children in Europe Programme. 2000 Dec-200I Jan;(2).

3. UNHCR NEWSLETTER. Separated Children in Europe Programme. 200I Feb-200I Mar;(3).

4. UNHCR NEWSLETTER. Separated Children in Europe Programme. 200I Oct-200I Nov;(9).

5. UNHCR NEWSLETTER. Separated Children in Europe Programme. 2002 Jun-2002 Jul;(II).

6. Anderson DL, Thompson GW, Popovich R (1977) Age of attainment of mineralization stages of the permanent dentition. Forensic Sci 2I(I): |9|-200.

7. Arany S, lino M, Yoshioka N (2004) Radiographic survey of third molar development in relation to chronological age among Japanese juveniles. J Forensic Sci 49(3):534-8

8. Aykroyd RG, Lucy D, Pollard AM, Solheim T (1997) Technical note: regression analysis in adult age assessment. Am J Phys Anthopol 104 259-265.

9. Bang G (1989) Age Changes in teeth: developmental and regressive. In: Iscan MY Age Markers in the Human Skeleton: 213. Charles C Thomas (ed). Springfield. Illinois.

10. Barbería E, De Nova J (2003) Maduración dental y determinación de la edad. La Determinación de la Edad en Detenidos Jóvenes Indocumentados. Problemática Actual y Protocolo de Valoración Médico-Forense: 31I-39. Segundo Curso de Actualización en Medicina Forense. Comunidad de Madrid.

II. Baume LJ, Becks H, Evans HM (1954) Hormonal control of tooth eruption. III. The response of the incisors of hypophysectomized rats to a growth hormone, thyroxin, or the combination of both. J Dent Res 33(I):104-I4.

I2. Blankenship JA, Mincer HH, Anderson KM, Woods MA, Burton EL (2007) Third molar development in the estimation of chronologic age in american blacks as compared with whites. J Forensic Sci 52(2):428-33.

13. Bolaños MV, Manrique MV, Bolaños MJ, Briones MT (2000) Approaches to chronological age assessment based on dental calcification. Forensic Sci Int II0:97-106.

14. Bolaños MV, Manrique MV, Bolaños M], Briones MT (2000) Determinación de la edad dental de los niños andaluces mediante el sistema de Demirjian. Ortodoncia Española 40(I):3I-8.

15. Bolaños Bolaños MV, Moussa H, Manrique MC, Bolaños MJ (2003) Radiographic evaluation of third molar development in Spanish children and young people. Forensic Sci Int I33(3):212-9. 16. Braga J, Heuze Y, Chavadel O, Sonan NK, Gueramy A (2005) Non-adult dental age assessment: correspondence analysis and linear regression versus Bayesian predictions. Int J Legal Med 119:260-274.

17. Burdi A.R. Desarrollo de la dentición y la oclusión.Moyers R.E. Manual de ortodoncia. Buenos Aires: Panamericana; 1992.
18. Convention on the Rights of the Child, G.A. res. 44/25, annex, 44 U.N. GAOR Supp. (No. 49) at I67, U.N. Doc. A/44/49 (1989), entered into force Sept. 2, 1990.

19. Davis PJ, Hagg U (1994) The accuracy and precision of the "Demirjian system" when used for age determination in Chinese children. Swed Dent J 18(3):II3-6.

20. Declaration of the Rights of the Child, G.A. res. I386 (XIV), I4 U.N. GAOR Supp. (No. 16) at 19, U.N. Doc. A/4354 (1959).

2I. Demirjian A. (1976) New systems for dental maturity based on seven and four teeth. Annals for Human Biology 3(5):4II-2I.

22. Demirjian A, Goldstein H, Tanner JM (1973) A new system of dental age assessment. Human Biology 42:211-27.

23. Dhanjal KS, Bhardwaj MK, Liversidge HM (2006) Reproducibility of radiographic stage assessment of third molars. Forensic Sci Int 15;159 Suppl I:S74-7.

24. Eid RM Simi R Friggi MN Fisberg M (2002) Assessment of dental maturity of Brazilian children aged 6 to years using Demirjian's method. Int J Paediatr Dent I2(6):423-8.

25. Evans KT, Knight B (198I) Radiology in forensic medicine. Oxford: Blackwell Scientific Publications.

26. Foti B, Lalys L, Adalian P, Giustiniani J, Maczel M, Signoli M et al (2003) New forensic approach to age determination in children based on tooth eruption. Forensic Sci Int I32(I):49-56.

27. Friedrich RE, Ulbricht C, von Maydell LA, Scheuer HA (2005) The impact of wisdom teeth topography on chronology of root formation--forensic consequence for forensic-odontologic age estimation of adolescents and young adults. Radiographic investigations using orthopantomography. Arch Kriminol 216(I-2):15-35.

28. Frucht S, Schnegelsberg C, Schulte-Monting J, Rose E, Jonas I (2000) Dental age in southwest Germany. A radiographic study. J Orofac Orthop 6I(5):318-29.

29. Fulton JT, Price B (1954) Longitudinal data on eruption and attack of the permanent teeth. J Dent Res 33:65-79.

30. Garamendi PM, Landa MI, Ballesteros J, Solano MA (2005) Reliability of the methods applied to assess age minority in living subjects around 18 years old. A survey on a Moroccan origin population. Forensic Sci Int 10;154(I):3-12.

31. Garn SM, Lewis AB (1962) Relationship between third molar agenesis and reduction in tooth number. Angle Orthodontist 32(I):14-8. 32. Garn SM, Lewis AB, Polachek DL (1959) Variability of Tooth Formation. J Dent Res 38:135-48.

33. Geneva Declaration of the Rights of the Child of 1924, adopted Sept. 26, 1924, League of Nations 0.J. Spec. Supp. 21, at 43 (1924). 34. German interdisciplinary working group for age diagnostics (200I) Recommendations on Age Diagnostics of Living Persons in Criminal Proceedings. Newsletter. German Association Of Forensic Odonto-Stomatology (AKFOS) 8(2).

35. Giles NB, Knott VB, Meredith HV (1963) Increase in intraoral height of selected permanent teeth during the quadrennium following gingival emergence. Angle Orthodontist 33:195-206. 
36. Giles E, Klepinger LL (1988) Confidence intervals for estimates based on linear regression in forensic anthropology. J. Forensic Sci 33:1218-1222.

37. Gorgani N, Sullivan RE, DuBois L (1990). ASDC J Dent Child 57(2):106-10.

38. Green L) (19619 The interrelationships among height, weight and chronological dental and skeletal ages. Angle Orthod 31:1890-6.

39. Gunst K, Mesotten K, Carbonez A, Willems G (2003) Third molar root development in relation to chronological age: a large sample sized retrospective study. Forensic Sci Int I36(I-3):52-7.

40. Haavikko K (1974) Tooth formation age stimated on a few selected teeth. A simple method for clinical use. Procc Finn Dent Soc 70:15-9.

4I. Hagg U, Taranger J (1980) Skeletal stages of the hand and wrist as indicators of the puberal growth. Acta Odontol Scand 38:187200.

42. Harris EF (2007) Mineralization of the mandibular third molar: a study of American blacks and whites. Am J Phys Anthropol. 2007 Jan;132(I):98-109.

43. Harris MJ, Nortjé CJ (1984) The mesial root of the third mandibular molar. A possible indicator of age. J Forensic Odontostomat 2(2):39-43.

44. Kieser JA (1990) Human Adults Odontometrics: The Study of Variation in Adult Tooth Size. New York: Cambridge University Press.

45. Köhler S, Schmelzle R, Loitz C, Püschel K (1994) Development of wisdom teeth as a criterion of age determination. Ann Anat 176(4):339-45.

46. Koshy S, Tandon S (1998) Dental age assessment: the applicability of Demirjian's method in south Indian children. Forensic Sci Int 94(I-2):73-85.

47. Kullman L, Johanson G, Åkesson L (1992) Root development of the lower third molar and its relation to chronological age. Swed Dent J 16:161-7.

48. Levesque GY, Demirjian A, Tanguay R (I98I) Sexual dimophism in the development, emergence and agenesis of the mandibular third molar. J Dent Res 60(10):1735-4I.

49. Lewis AB, Garn SM (1960) The relationship between tooth formation and other maturational factors. Angle Orthod 70:70-7.

50. Ley Orgánica 5/2000, of 12 January, regulating legal responsibility of minors. BOE n⿳II I3-I-2000: 1422-I44I

51. Liversidge HM (1994) Accuracy of age stimation from developing teeth of a population of known age (0 to 5,4 years). Int Journal Osteoarchaeol 4:37-46.

52. Liversidge HM, Speechly T, Hector MP (1999) Dental maduration in British children: are Demirjian's standars applicable. Int J Pediatr Dent 9:263-9.

53. Loevy HT, Goldberg AF (1999) Shifts in tooth maturation patterns in non-French Canadian boys. Int J Pediatr Dent 9(2):105-10. 54. Martín C. Bernabeu D. Maduración Osea. Comunidad de Madrid. Dirección General de Justicia. Segundo Curso de Actualización en
Medicina Forense. La Determinación de la Edad en Detenidos Jóvenes Indocumentados. Problemática Actual y Protocolo de Valoración Médico-Forense.2003. pp. 27I-309.

55. Martin-de Las Heras S, García-Fortea P, Ortega A, Zodocovich S, Valenzuela A (2007) Third molar development according to chronological age in populations from Spanish and Magrebian origin. Forensic Sci Int 23 (ahead of print). Forensic Sci Int. 2008 Jan I5;|174(I):47-53.

56. Mckenna CJ, James H, Taylor JA, Townsend GC (2002) Tooth development standards for South Australia. Aust Dent J 47(3):223-7.

57. Meinl A, TangI S, Huber C, Maurer B, Watzek G (2007) The chronology of third molar mineralization in the Austrian population--a contribution to forensic age assessment. Forensic Sci Int 169(2-3):161-7.

58. Mesotten K, Gunst K, Carbonez A, Willems G (2002) Dental age assessment and third molars: a preliminary study. Forensic Sci Int 129:110-5.

59. Micci M, Buzzanca $R(1998)$ Age and mineralization of third molars in a group from Enna and Caltonissetta provinces. Stomatol Mediterr 8(2):123-5.

60. Mincer HH, Harris EF, Berryman HE (1993) The A.B.F.O. study of third molar development and its use as an estimator of chronological age. J For Sci 38(2):379-90.

61. Moorrees CFA, Fanning EA, Hunt EE (1963) Formation and resorption of three deciduous teeth in children. Am J Phys Anthropol 21:99-108.

62. Morrees CFA, Fanning EA, Hunt EE (1963) Age variation of formation stages for ten permanent teeth. J Dent Res 42(6):149-502. 63. Moorrees C.F. Kent R.L. Patterns of Dental Maturation. The Biology of Occlusal Development.1977. pp. 25-4I.

64. Nambiar P (1996) Third molars in the stablisment of adult status. A case report. J Forensic Odontostomatol I4(2):30-3.

65. Nolla CM (1960) The development of the permanent teeth. J Dent Child 27:254-66.

66. Nykanen R, Espeland L, Kvaal SI, Krogstad 0 (1998) Validity of Demirjian method for dental age assessment when applied to Norwegian children. Acta Odontol Scand 56(4):238-44.

67. Nystrom M, Haataja J, Kataja M, Evalahti M, Peck L, KleemolaKujala $E$ (1986) Dental maturity in finnish children, estimated from the development of seven permanent mandibular teeth. Acta Odontol Scand 44(4):193-8.

68. Olze A, Schmeling A, Taniguchi M, Maeda H, van Niekerk P, Wernecke KD, Geserick $G$ (2004) Forensic age estimation in living subjects: the ethnic factor in wisdom tooth mineralization. Int J Legal Med II8(3):170-3.

69. Olze A, Bilang D, Schmidt S, Wernecke KD, Geserick G, Schmeling A (2005) Validation of common classification systems for assessing the mineralization of third molars. Int J Legal Med II9(I):22-6.

70. Olze A, Reisinger W, Geserick G, Schmeling A (2006) Age estimation of unaccompanied minors. Part II. Dental aspects. Forensic Sci Int 159 Suppl I:S65-7. 
7I. Olze A, van Niekerk P, Schmidt S, Wernecke KD, Rosing FW, Geserick G, Schmeling A (2006) Studies on the progress of thirdmolar mineralisation in a Black African population. Homo 57(3):209-17.

72. Olze A, van Niekerk P, Ishikawa T, Zhu BL, Schulz R, Maeda H, Schmeling A (2007) Comparative study on the effect of ethnicity on wisdom tooth eruption. Int J Legal Med Apr: 24 (ahead of print).

73. Orhan K, Ozer L, Orhan Al, Dogan S, Paksoy CS (2007) Radiographic evaluation of third molar development in relation to chronological age among Turkish children and youth. Forensic Sci Int I65(I):46-5I.

74. Planells P, De Nova J, Barbería E (1993) Cronología de la erupción dentaria II. Comparación entre arcadas. Rev Iberoamer de Ortod I2(I):4I-9.

75. Prabhakar AR, Panda AK, Raju OS (2002) Applicability of Demirjian's method of age assessment in children of Davangere. Indian Soc Pedod Prev Dent 20(2):54-62.

76. Prieto LL, Barbería E, Ortega R, Magaña C (2007) Evaluation of chronological age based on third molar development in the Spanish population. Int J Legal Med II9(6):349-54.

77. Ramirez 0, Planells P, Barberia E (1994) Age and order of eruption of primary teeth in Spanish children. Community Dent Oral Epidemiol 22(I):56-9.

78. Ritz-Timme S, Cattaneo C, Collins MJ, Waite ER, Schutz HW, Kaatsch HJ, Borrman HI (2000) Age estimation: the state of the art in relation to the specific demands of forensic practise. Int ] Legal Med II3(3):I29-36.

79. Robetti I, lorio M, Guglielmetti Mugion T (1982) Age of third molar mineralization. A bibliographic review and synoptic table. Minerva Stomatol 3I(6):837-9.

80. Robetti I. Iorio M, Dalle Molle M (1993) Orthopantomography and the determination of majority age. Panminerva Med 35:3.

8I. Saunders S. De Vito C. Herrig A. Southern R. Hoppa R (1993) Accuracy tests of tooth formation age estimations for human skeletal remains. Am J Phys Anthropol 92:173-88.

82. Savara BS (1978) Timing and sequence of eruption of permanent teeth in a longitudinal sample of children from Oregon. JADA 97:209-14.

83. Schmeling A, Olze A, Reisinger W, Geserick G (2004) Forensic age diagnostics of living people undergoing criminal proceedings. Forensic Sci Int I44(2-3):243-5.
84. Schmeling A, Geserick G, Reisinger W, Olze A (2007) Age estimation. Forensic Sci Int I65(2-3):178-8I.

85. Solari AC, Abramovitch K (2002) The Accuracy and Precision of Third MOlar Development as an Indicator of Chronological Age in Hispanics. J Forensic Sci 47(3):53I-5.

86. Solheim T, Vonen A (2006) Dental age estimation, quality assurance and age estimation of asylum seekers in Norway. Forensic Sci Int I59 Suppl I:S56-60.

87. Staaf V, Mönstard H, Welander U (199I) Age stimation based on tooth development: a test of reliability and validity. Scand J Dent Res 99:28I-6.

88. Tanguay R, Demirjian A, Thilbaut HW (1984) Sexual dimorphism in the emergence of deciduous teeth. J Dent Res 63(I):65-8.

89. Tanner JM, Whitehouse RM, Marshall WA, Healy MJR, Goldstein H (1975) Assessment of skeletal maturity and prediction of adult height TW2 method. Academic Press. London.

90. Teivens A, Mörnstad H (200I) A modification of the Demirjian method for age stimation in children. J Forensic Odontostomatol 19(2):26-30.

91. Thorson J, Hagg U (199I) The accuracy and precision of the third mandibular molar as an indicator of chronological age. Swed Dent J I5(I):15-22.

92. Ubelaker DH (1999) Human skeletal remains. Excavation,

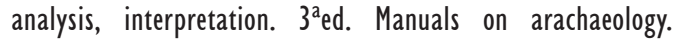
Taraxacum. Washington.

93. Ungar AL (1937) Incidence and effect of premature loss of deciduous teeth. Int J Orthod 24:613-25.

94. Van Der Linden F (1980) La transición de la dentición humana. Rev Esp Ort 10:1-96.

95. Willems G, Van Olmen A, Spiessens B, Carels C (200I) Dental age assessment in Belgian children: Demmirjian's technique revisited. J Forensic Sci 46(4):893-5.

96. Willershausen B, Loffler N, Schulze R (200I) Analysis of I202 orthopantomograms to evaluate the potential of forensic age determination based on third molar development stages. Eur J Med Res 28(6):377-84.

97. Yaacob H, Nambiar P, Naidu MD (1996) Racial characteristics of human teeth with special emphasis on the Mongoloid dentition. Malays J Pathol I8(I):I-7. 